\title{
Intensity noise in ultra-high frequency modulated semiconductor laser with strong feedback and its influence on noise figure of RoF links
}

M. Ahmed

mostafa.hafez@science.miniauniv.edu.eg

A. Bakry

R. Altuwirqi

M. S. Alghamdi

F. Koyama

koyama@pi.titech.ac.jp

\begin{abstract}
Department of Physics, Faculty of Science, King Abdulaziz University, 80203 Jeddah 21589, Saudi Arabia.

Department of Physics, Faculty of Science, King Abdulaziz University, 80203 Jeddah 21589, Saudi Arabia.

Department of Physics, Faculty of Science, King Abdulaziz University, 80203 Jeddah 21589, Saudi Arabia.

Department of Physics, Faculty of Science, King Abdulaziz University, 80203 Jeddah 21589, Saudi Arabia.

Department of Physics, Faculty of Science, King Abdulaziz University, 80203 Jeddah 21589, Saudi Arabia.
\end{abstract}

Photonics Integration System Research Center, Precision and Intelligence Laboratory, Tokyo Institute of Technology, Yokohama 226-8503, Japan.

We characterize the intensity noise associated with the ultra-high frequency direct modulation of semiconductor lasers under strong optical feedback over a millimeter-wave frequency passband between 54.5 and $56.5 \mathrm{CHz}$. Enhancement of the modulation response over this millimeter wave band due to optical feedback is clearly shown by time-delay rate equation analysis fully involving the strong optical feedback effect. The contribution of the relative intensity noise of the laser to the noise figure of an ultra-high speed radio over fiber link is evaluated. We show that subjecting the laser diode to strong feedback improves the noise figure of a 55.8- $\mathrm{CHz}$ radio over fiber link nearly by $20 \mathrm{~dB}$ in the regime of small-signal modulation and $10 \mathrm{~dB}$ under large-signal modulation.

[DOI: http://dx.doi.org/10.2971/jeos.2013.13064]

Keywords: Modulation, intensity noise, optical feedback, RoF link, semiconductor laser

\section{INTRODUCTION}

The radio over fiber (RoF) technology has been emerged as an integration of wireless and optical systems to provide integrated broadband services in wireless local area networks (WLANs) [1]. Such broadband services require enhancing the bandwidth of RoF links to the mm-wave band, which necessitates boosting the modulation bandwidth of directlymodulated laser diodes in RoF links. It is well-known that the modulation bandwidth of semiconductor lasers is limited by their relaxation oscillation frequencies, which makes a difficulty in direct modulations in mm-wave bands. The modulation bandwidth of lasers can be enhanced by injection locking [2, 3]; high-frequency modulation beyond $40 \mathrm{GHz}$ was demonstrated $[4,5]$. However, a complex configuration is needed in this scheme. External optical feedback (OFB) has been also reported as an alternative and cost-effective technique to increase the modulation bandwidth of semiconductor laser, depending on appropriate choices of the system parameters [6]-[11]. Narrow-band high-frequency modulation over $40 \mathrm{GHz}$ has been achieved in quantum well lasers under OFB [12]. The authors have reported on using strong OFB to boost the modulation frequencies over an ultra-high frequency passband over $50 \mathrm{GHz}$ and have shown improvement of the link gain nearly by $20 \mathrm{~dB}$ over using solitary lasers [13]. On the other hand, noise is a primary factor affecting efficiency of RoF links. It is desirable that the smaller is the noise factor and the higher is the efficiency of the link [14]. The intensity noise of semiconductor lasers is caused by random fluctuations in carrier and photon recombination and generation processes, which result in instantaneous time variations in the carrier and photon densities [15]. Under direct modulation, the laser is preferably operated in the vicinity of the relaxation frequency where the modulation response is maximum. However, due to the complicated nonlinear effects in this regime, the laser noise increases specially under large modulation depth [16]. On the other hand, the application of external OFB to the laser diode may amplify its intrinsic fluctuations and deteriorate its noise performance, depending on the system parameters [17]-[19]. When OFB is applied to a laser diode, the laser noise decreases below the quantum level under very weak $\mathrm{OFB}$, and then increases with the increase of OFB along a route-to-chaos [19]. The noise is then enhanced in the intermediate range of OFB where the laser becomes chaotic and coherently collapsed [17, 19]. In the regime of strong OFB, laser noise exceeds the quantum limit and its level depends on whether the laser operates in CW or emits uniform/non-uniform oscillations [19]. Study of laser noise under both OFB and intensity modulation has been studied by several groups specially for controlling OFB-noise by modulating the laser in the vicinity of the relaxation oscillations [20]-[22]. However, studying the noise problem at 
modulation frequencies much higher than the relaxation frequency, such as the case of ultra-high frequency passband, has not been reported. In this paper, we apply a time-delay rate equation model [23] to study the intensity noise associated with ultra-high frequency modulation of semiconductor lasers under strong OFB. We also evaluate the contribution of this noise to the noise performance of a designed RoF link in terms of its noise figure (NF). The applied OFB model treats OFB as time delay of laser radiation due to roundtrips (multiple reflections) in the external cavity formed between the front facet of the laser and the external reflector [23]. The model enables us to treat the strong OFB regime. The laser noise is characterized by the frequency spectrum of the relative intensity noise (RIN) and its level in the low-frequency regime, LFRIN. We compared the obtained findings with those of a solitary laser when modulated at the same modulation frequency and within the vicinity of the relaxation oscillation frequency as well. We apply the model to a high-speed DFB laser with a modulation bandwidth of about $25 \mathrm{GHz}$ [24]. We show that by adopting the distance of the external reflector to correspond to an external-cavity resonance frequency of about $60 \mathrm{GHz}$, a modulation frequency band of about $2 \mathrm{GHz}$ around a frequency of $55 \mathrm{GHz}$ can be achieved by strong OFB. We show also that NF increases from 4 to $34 \mathrm{~dB}$ with the increase in the modulation depth from 0.05 to 1 , and that NF improves by using OFB nearly by $20 \mathrm{~dB}$ in the regime of small-signal modulation and $10 \mathrm{~dB}$ under large-signal modulation.

\section{THEORETICAL MODEL}

We describe the dynamics and modulation characteristics of semiconductor lasers under external OFB by the following time-delay rate equations of the carrier density $N(t)$, photon density $S(t)$ and optical phase $\theta(t)$

$$
\begin{gathered}
\frac{d N}{d t}=\frac{1}{e V} I(t)-\frac{N}{\tau_{s}}-a v_{g} \frac{N-N_{g}}{1+\epsilon S}+F_{N}(t) \\
\frac{d S}{d t}=\left[\Gamma a v_{g} \frac{N-N_{g}}{1+\epsilon S}-\frac{1}{\tau_{p}}+\frac{v_{g}}{L_{D}} \ln |U|\right] S+C \frac{N}{\tau_{s}}+F_{S}(t) \\
\frac{d \theta}{d t}=\frac{1}{2}\left(\alpha \operatorname{\Gamma av}_{g} \frac{N-N_{g}}{1+\epsilon S}-\frac{v_{g}}{L_{D}} \phi\right)+F_{\theta}(t)
\end{gathered}
$$

This model of OFB is generalization of the Lang and Kobayashi model [25] by taking into account both the strong OFB regime and the multiple reflections in the external cavity [23]. $U$ is an OFB function that describes the time delay of laser radiation due to roundtrips (i.e., multiple reflections) in the external cavity formed between the laser front facet (of reflectivity $\mathrm{Rf})$ and the external mirror (Rex) [23],

$$
\begin{aligned}
U & =|U| e^{-j \phi} \\
& =1-\sum_{p=1}\left(K_{e x}\right)^{p}\left(\frac{R_{f}}{1-R_{f}}\right)^{p-1} e^{-j p \omega \tau} \frac{S(t-p t)}{S(t)} \frac{e^{j \theta(t-p \tau)}}{e^{j \theta(t)}}
\end{aligned}
$$

with $\omega$ being the angular frequency of the laser emission and $\tau=2 n_{e x} L_{e x} / c$ as the roundtrip time, where $n_{e x}$ and $L_{e x}$ are the refractive index and length of the external cavity, respectively, and $c$ is the speed of light in vacuum. The strength of OFB is

\begin{tabular}{|c|c|c|}
\hline Symbol & Definition & Value \\
\hline$\lambda$ & Wavelength & $1.55 \mu \mathrm{m}$ \\
$V$ & Active layer volume & $3 \times 10^{-11} \mathrm{~cm}^{3}$ \\
$v_{g}$ & Group velocity & $8.33 \times 10^{9} \mathrm{~cm} / \mathrm{s}$ \\
$L_{D}$ & Active layer length & $120 \mu \mathrm{m}$ \\
$\eta_{0}$ & Quantum efficiency & 0.255 \\
$a_{0}$ & Differential gain coefficient & $9.9 \times 10^{-16} \mathrm{~cm}^{2}$ \\
$N_{g}$ & Carrier density at transparency & $1.23 \times 10^{18} \mathrm{~cm}^{-3}$ \\
$\alpha$ & Linewidth enhancement factor & 3.5 \\
$\Gamma$ & Mode confinement factor & 0.2 \\
$\tau_{s}$ & Electron lifetime due to & $0.766 \times 10^{-9} \mathrm{~s}$ \\
$R_{f}$ & Spontaneous emission & 0.2 \\
$R_{b}$ & Front facet reflectivity & 0.6 \\
$\tau_{p}$ & Back facet reflectivity & $1.69 \times 10^{-12} \mathrm{~s}$ \\
$\beta_{s p}$ & Ppontaneous emission factor & $3 \times 10^{-5}$ \\
$\epsilon$ & Nonlinear gain suppression & $2.77 \times 10^{-17} \mathrm{~cm}^{3}$ \\
\hline
\end{tabular}

TABLE 1 Definition and numerical values of the solitary high-speed laser parameters.

figured by the coupling coefficient $K_{e x}$, which is determined by the ratio between $R_{e x}$ and $R_{f}$,

$$
K_{e x}=\left(1-R_{f}\right) \sqrt{\eta \frac{R_{e x}}{R_{f}}}
$$

where $\eta$ is the external coupling efficiency of the injected light into the laser cavity. The injection current is composed of a bias component $I_{b}$, and a modulation component characterized by modulation current $I_{m}$ and frequency $f_{m}$

$$
I(t)=I_{s}+I_{m} \sin \left(2 \pi f_{m} t\right)
$$

Both $I_{b}$ and $I_{m}$ define the modulation depth $m=I_{m} / I_{b}$. The laser parameters appeared in the above equations are defined in Table 1 . The last terms $F_{N}(t), F_{S}(t)$ and $F_{\theta}(t)$ in rate Eqs. (1)-(3) are Langevin noise sources with zero mean values, and are added to the equations to account for intrinsic fluctuations of the laser [15]. These noise sources are assumed to have Gaussian probability distributions and to be $\delta$-correlated processes [15]. The frequency content of intensity fluctuations is measured in terms of RIN, which is calculated from the fluctuations $\delta S(t)=S(t)-S_{b}$ in $S(t)$, where $S_{b}$ is the bias value of $S(t)$. Over a finite time $T$, RIN is given as [15]

$$
R I N=\frac{1}{S_{b}^{2}}\left\{\frac{1}{T}\left|\int_{0}^{T} \delta S(t) e^{j 2 \pi f t} d \tau\right|^{2}\right\}
$$

where $f$ is the Fourier frequency. The noise performance of the laser is evaluated also in terms of the average value of the RIN components at frequencies lower than $100 \mathrm{MHz}, \mathrm{LF}-\mathrm{RIN}$. It is worth noting that according to Eq. (7), the RIN spectrum includes not only the noise but also the signal power spectral density. In this way, we are able to include the effects of nonlinear mixing between the fundamental noise and the signal whereas RIN is conventionally calculated and measured without any modulation using a small signal approximation. 


\section{NUMERICAL CALCULATIONS}

Rate Eqs. (1)-(3) are solved by the $4^{\text {th }}$ order Runge-Kutta method using a time integration step as short as 0.2 ps to allow simulation of the very-high speed modulated signal. Five roundtrips ( $p=1 \rightarrow 5$ in Eq. (4)), each of duration of $\tau$, are counted in the calculations, which is large enough to take into account multiple reflections occurring in the external cavity. First, the integration is solved without OFB from time $t=0$ until the first round trip time $\tau$. The calculated values of $S$ and $\theta$ are then stored for use as time delayed values $S(t-\tau)$ and $\theta(t-\tau)$ for integration of the rate equations over the period $t=\tau \rightarrow 2 \tau$ including the OFB terms. Then the calculated values $S(t-\tau), S(t-2 \tau), \theta(t-\tau)$ and $\theta(t-\tau)$ are used as time delayed values for integration over the period. This process continues up to the $5^{\text {th }}$ roundtrip. The integration is then proceeded over a long period of time $T=2.5 \mu$ s considering all terms of $S(t-p \tau)$ and $\theta(t-p \tau)$ as time delayed values. Both the modulation response and spectra of RIN are calculated over the time period from 2 to $2.5 \mu$ s over which the laser output is stable. At each integration instant, the noise sources $F_{N}(t), F_{S}(t)$ and $F_{\theta}(t)$ are generated following the technique in [26] using a set of uniformly distributed random numbers generated by the computer. In the simulations, we use the numerical values listed in Table 1 that correspond to a highspeed strained quantum-well DFB laser [24]. This laser has a threshold current of $I_{t h}=10 \mathrm{~mA}$ and, as we will show, a relaxation frequency of $f_{r}=14 \mathrm{GHz}$. The laser is assumed to be biased far above threshold, $I_{b}=5 I_{t h}$. This injection level is high enough to reduce contribution of the spontaneous emission to the laser output, which is significant near the laser threshold [15]. This high injection level would help also to reduce the intrinsic noise level of the laser and gain insight into the noise induced by to both intensity modulation and OFB. We adapt the length of the external cavity to be $n_{e x} L_{e x}=0.25 \mathrm{~cm}$, which corresponds to an external-cavity resonance frequency spacing $60 \mathrm{GHz}$. Either an external cavity or monolithic extended cavity [11] can be considered in this model.

\section{RESULTS AND DISCUSSION}

The present case of a semiconductor laser with a short cavity is characterized by a frequency ratio $f_{e x} / f_{r}>1$, which corresponds to a period-doubling route-to-chaos $[27,28]$. The frequency of possible oscillations under strong OFB increases with the increase in the OFB strength, starting from the relaxation oscillation of the laser $f_{r}$ toward the external-cavity resonance frequency $f_{e x}=c /\left(2 n_{e x} L_{e x}\right)=60 \mathrm{GHz}$ under strong OFB. In the regime of strong OFB, we simulate the laser modulation under OFB with the level of $K_{e x}=1.45$ at which the solitary laser operation is stable CW. Figure 1 plots the simulated modulation response for modulation depth $m=0.1$ and plots also the modulation response of the solitary laser for comparison. The figure shows that the modulation response of the solitary laser is a maximum when $f_{m}=14 \mathrm{GHz}$ and has a 3dB-modulation bandwidth of $f_{3 \mathrm{~dB}}=25 \mathrm{GHz}$. Under the strong OFB level of $K_{e x}=1.45$, the modulation response exhibits the interesting feature that it drops under the $-3 \mathrm{~dB}$ level at the modulation frequency of $14.5 \mathrm{GHz}$, which is much lower than $f_{3 \mathrm{~dB}}$ of the solitary laser, and en-

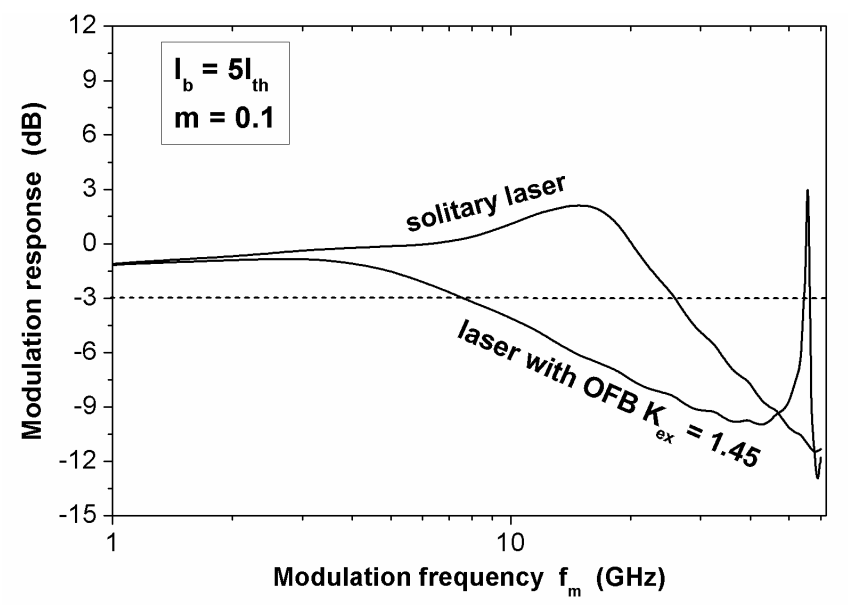

FIG. 1 Numerical modulation responses of both the solitary laser and laser under strong OFB $K_{e x}=1.45$, when $I_{b}=5 I_{t h}$ and $m=0.1$.

hances again over an ultra-high narrow frequency band of (54.5 56.5 GHz). This frequency band is much higher than $f_{3 \mathrm{~dB}}$ of the solitary laser, which enables us to modulate in mm-wave high-frequency bands. This enhanced modulation response is manifestation of a resonance nearly at the external cavity resonance frequency $f_{e x}$, and can be also interpreted as one (or both) of the modulation sidebands of the modulated laser coincides with an optical side mode of the cavity and is resonantly amplified. Figure 1 indicates also that at the ultra-high frequency $f_{m}=55.8 \mathrm{GHz}$, the modulation response under strong OFB is almost $12 \mathrm{~dB}$ higher than that of the solitary laser. It is important to note that both the optical phase $\theta(t)$ and the OFB phase $\theta(t-\tau)$ are functions of time and are coupled to the instantaneous variations in both $S(t)$ and $N(t)$ through the argument $\phi$ of the feedback function $U$. Since the optical phase of the feedback field is sensitive to temperature and mechanical variations, we investigate the influence of this coupling phase on the modulation response of the laser by assuming steady state OFB phase. The OFB coupling phase $\Delta \theta=\theta(t)-\theta(t-\tau)$ is set to be constant values ranging between zero and $2 \pi$. Figure 2 plots the small modulation responses simulated at different values of $\Delta \theta$ with neglecting the frequency chirping. The figure shows that the modulation response enhancement beyond $50 \mathrm{GHz}$ occurs when $\pi \leq \Delta \theta \leq 1.5 \pi$. The figure indicates also that the maximum response enhancement is obtained when $\Delta \theta=1.3 \pi$, and the peaked modulation frequency of the response enhancement increases with the increase in the OFB coupling phase $\Delta \theta$. Enhancement of the modulation bandwidth under out-ofphase coupling feedback was reported by Koyama and Dalir [29] in VCSELs using a small-signal modulation analysis in a weak-coupling regime. Thus it is noted that the phase of optical feedback should be controlled for getting the modulation response enhancement over the $\mathrm{mm}$ wave band. In Figure 3, we explore the spectral characteristics of RIN of the modulated laser under strong OFB. The figure plots also the frequency spectra of RIN of the solitary laser when modulated at the same ultra-high frequency $f_{m}=55.8 \mathrm{GHz}$ and at $f_{m}=15 \mathrm{GHz}$ in the vicinity of the maximum modulation response. The modulated signal is clearly seen in these RIN spectra. The Fourier frequency $f$ of the horizontal axis of the figure is normalized by the modulation frequency $f_{m}$ of each 


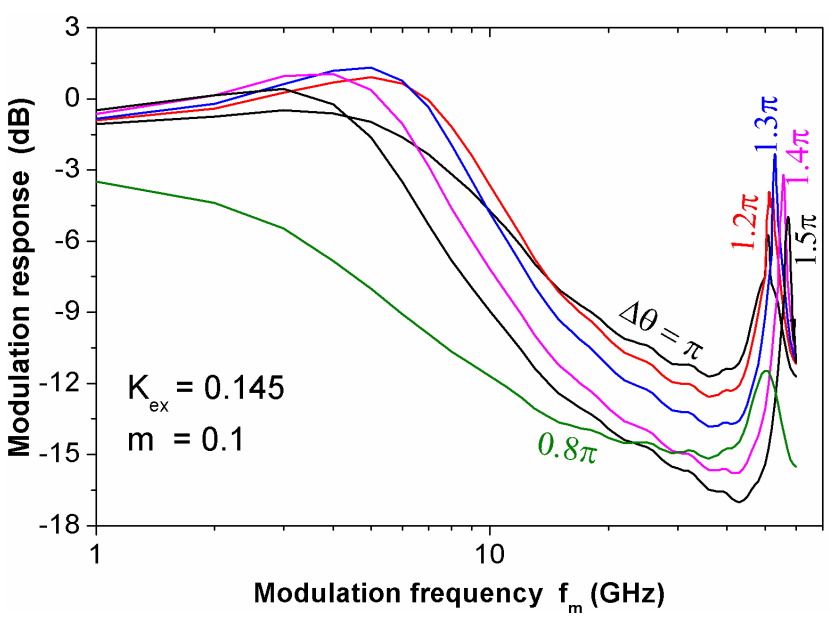

FIG. 2 Modulation responses at different values of the constant OFB coupling phase $\Delta \theta$ when $K_{e x}=1.45$ and $m=0.1$.

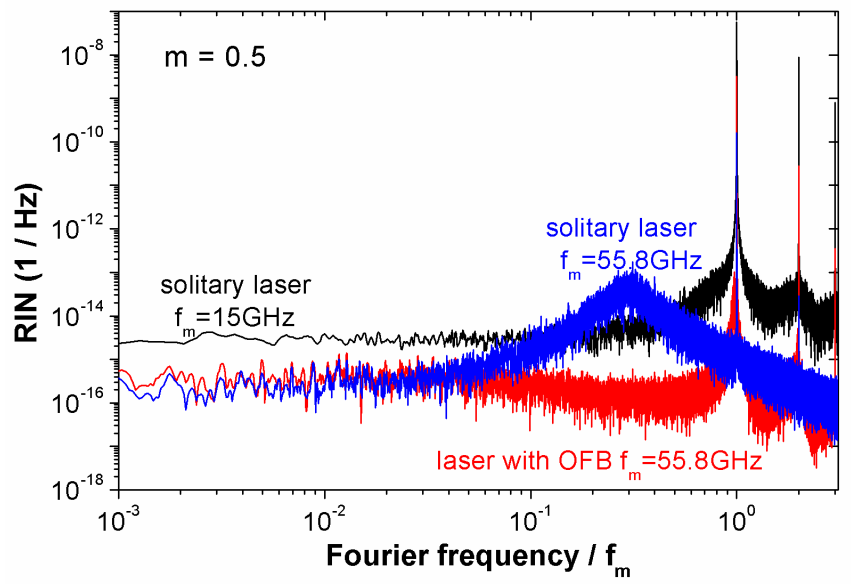

FIC. 3 Frequency spectra of RIN of the laser under OFB when modulated with $f_{m}=55.8 \mathrm{GHz}$ and the solitary laser when modulated with $f_{m}=15$ and $55.8 \mathrm{CHz}$. $m=0.5$.

signal to compare the modulation characteristics of the relevant signals. The figure shows that the RIN spectra have sharp peaks at the corresponding modulation frequency $\mathrm{fm}$ and at the higher harmonics. These peaks are most pronounced for the laser under OFB and weakest for the $55.8 \mathrm{GHz}$-modulated solitary laser. The latter exhibits also a peak around the relaxation frequency $f_{r}$ of the solitary laser, which is because the modulation response of the solitary laser at this ultra-high frequency is comparable to the amplitude of quantum fluctuations. The LF-RIN level of the laser under OFB is three times lower than that of the $15 \mathrm{GHz}$-modulated solitary laser and is little higher than that of the $55.8 \mathrm{GHz}$-modulated solitary laser. In Figure 4, we compare the LF-RIN level of the three cases over a wide range of the modulation depth $m$. The figure shows that LF-RIN increases very little with the increase in $\mathrm{m}$ for both the laser under OFB and the solitary laser when modulated at $f_{m}=55.8 \mathrm{GHz}$. On the other hand, LF-RIN of the solitary laser modulated at $f_{m}=15 \mathrm{GHz}$ increases considerably with the increase in $m$ due to an increase in the degree of distortion in the modulated signal near the relaxation frequency [16]. This distortion causes redistribution of the optical power from the fundamental tone to the harmonics and is associated with nonlinear down-conversion of high frequency

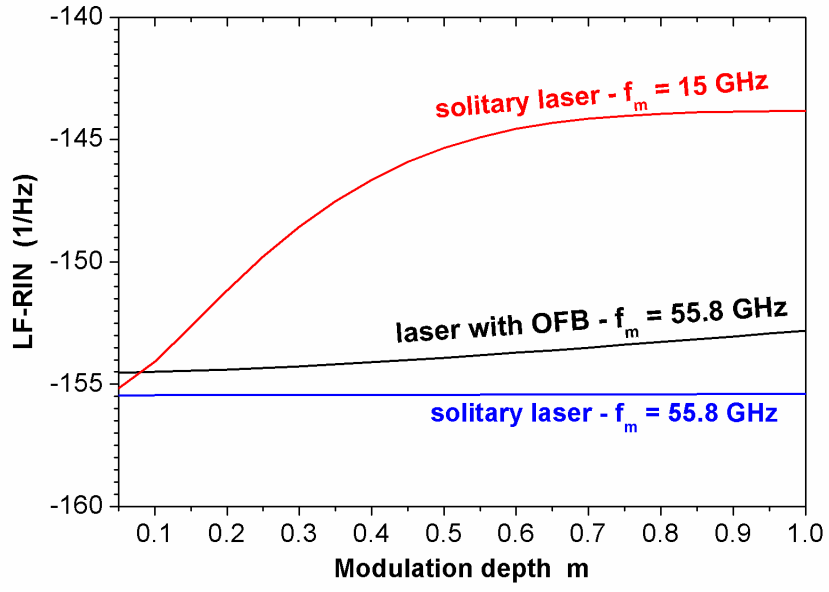

FIG. 4 LF-RIN as a function of modulation depth $m$ of the laser under OFB when modulated with $f_{m}=55.8 \mathrm{CHz}$ and the solitary laser when modulated with $f_{m}=15$ and $55.8 \mathrm{GHz}$

noise. To assess the contribution of the laser RIN to the noise performance of ultra-high speed RoF links, we assume using the laser under OFB to design of a lossy RoF link along with a short fiber $(300 \mathrm{~m})$ and a PIN photodiode. The efficiency of the link is evaluated in terms of the link gain $G_{l i n k}$, and the noise performance is measured by the noise figure NF. For such calculations, we apply the model proposed by Ackerman and Cox [30] and Cox [31] in which two lossless transformer impedance-matching circuits are supposed to match the laser and PIN impedances to the RF source and load impedances, respectively. $G_{\text {link }}$ is then given as

$$
\begin{aligned}
G_{\text {link }} & =\left(\frac{P_{L}^{2}}{P_{S}}\right)\left(T_{L-D}\right)^{2}\left(\frac{P_{\text {load }}}{P_{D}^{2}}\right) \\
& =\frac{S_{L}^{2} N_{L}^{2}}{R_{S}\left[\left(\pi f_{m} C_{L} R_{L}\right)+1\right]^{2}} T_{L-D}^{2} \times \frac{r_{d}^{2} N_{D}^{2} R_{\text {load }}}{\left(2 \pi f_{m} C_{D}\right)^{2}\left(R_{D}+N_{D}^{2} R_{\text {load }}\right)^{2}+1}
\end{aligned}
$$

where $P_{L}$ is the optical power delivered by the laser diode of slope efficiency $S_{L}, P_{S}$ is RF-power available at the input modulating RF source at a given modulation frequency $f_{m}, P_{D}$ is the RF-power delivered by the PIN photodiode of responsivity $r_{D}$, and $P_{\text {load }}$ is the RF-power delivered to a matching load resistance $R_{\text {load }}$. $T_{L-D}$ is the transmission of the laser signal from the laser diode to the PIN photodiode including the fiber loss $\alpha_{\text {fiber }}$ and both the coupling ratios between the laser and fiber $\eta_{L-F}$ and between the fiber and PIN photodiode $\eta F-D$. The noise figure NF is then given in terms of $G_{\text {link }}$ and the receiver bandwidth $B$ at temperature $T$ as [31]

$$
N F(\mathrm{~dB})=10 \log _{10}\left(\frac{N_{\text {out,RIN }}+N_{\text {out,shot }}+N_{\text {out,thermal }}}{k T B G_{\text {link }}}\right)
$$

where $N_{\text {out,RIN }}, N_{\text {out,thermal }}$ and $N_{\text {out,shot }}$ are the electrical power dissipations caused by noise currents in the load resistance due to the laser noise, and the thermal and shot noises in the photodetector, respectively. These noises are given by [31]

$$
\begin{gathered}
N_{\text {out }, \text { RIN }}=\left\langle i_{\text {RIN }}^{2}\right\rangle R_{\text {load }}=R_{\text {load }} I_{\text {ph }}^{2} B R I N \\
N_{\text {out,shot }}=\left\langle i_{\text {shot }}^{2}\right\rangle R_{\text {load }}=2 q R_{\text {load }} B\left(I_{\text {ph }}+I_{d}\right) \\
N_{\text {out,thermal }}=\left\langle i_{\text {thermal }}^{2}\right\rangle R_{\text {load }}=4 k B T
\end{gathered}
$$




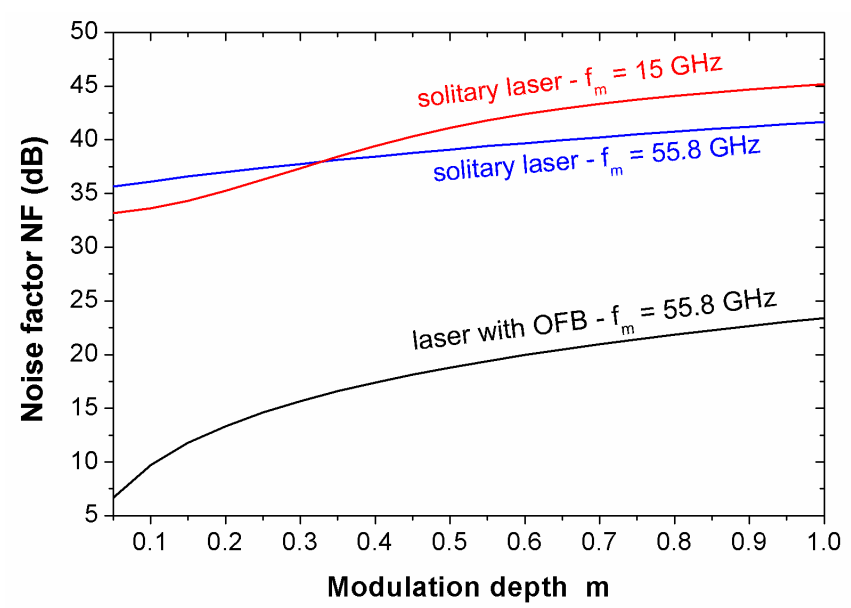

FIG. 5 Noise factor as a function of modulation depth $m$ of the laser under OFB when modulated with $f_{m}=55.8 \mathrm{GHz}$ and the solitary laser when modulated with $f_{m}=15$ and $55.8 \mathrm{GHz}$.

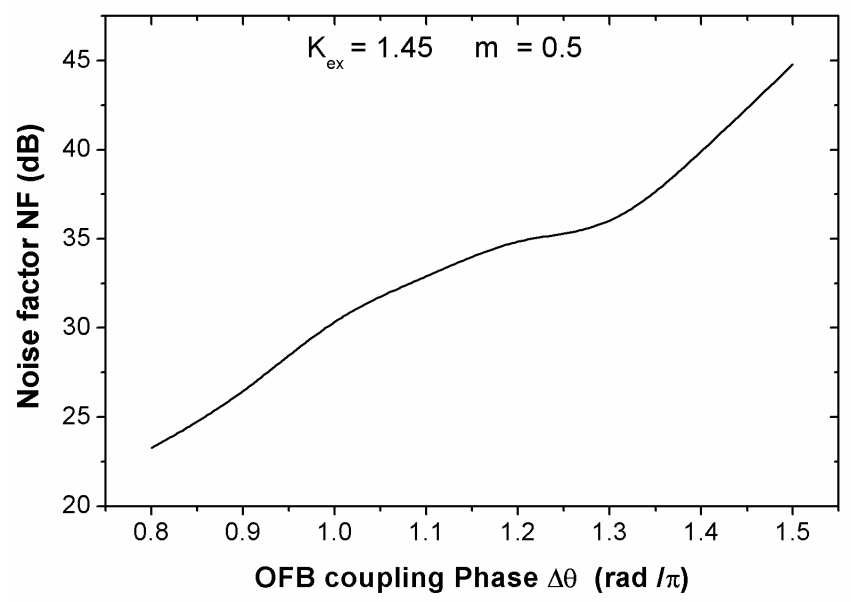

FIG. 6 Noise factor as a function of the OFB coupling phase $\Delta \theta$ when $K_{e x}=1.45$ and $m=0.5$.

In the above equations $I_{p h}=r_{D} T_{L-D} P_{L}$ and $I_{d}$ are the average DC photocurrent and dark current in the photodetector. Other parameters and their values assigned for RF links with bandwidth exceeding $60 \mathrm{GHz}$ include $R_{S}=50 \Omega$ as a series diode resistance, $R_{L}=2 \Omega$ and $R_{\text {load }}=20 \Omega$ as load resistances at the transmitter and receiver, respectively, $R_{D}=2 \Omega$ as the phorodiode series resistance, $C_{L}=1 p F$ and $C_{D}=0.1 p F$ as laser and photodiode junction capacitances, respectively, and $N_{L}=5$ and $N_{D}=1.7$ as the turns-ratio of transformer twists at the transmitter and receiver, respectively. In the present calculations, we consider the white RIN, LF-RIN, in the frequency band below the relaxation frequency $f_{r}$. In this case, NF becomes independent of the frequency bandwidth $B$ because both the thermal and shot noises are white [30]. In Figure 5, we plot the calculated values of NF as a function of the modulation depth $m$ for the laser under strong OFB with $f_{m}=55.8 \mathrm{GHz}$ and the solitary laser modulated at $f_{m}=15$ and $55.8 \mathrm{GHz}$. The figure shows that NF of the $55.8 \mathrm{GHz}-\mathrm{RoF}$ link with laser under strong OFB increases from 4 to $34 \mathrm{~dB}$ with the increase in $m$ from 0.05 to 1 . On the other hand, NF of the link using the solitary laser ranges between 33 and 42 $\mathrm{dB}$. As indicated by the figure, subjecting the laser diode to strong OFB improves NF of the $55.8 \mathrm{GHz}-\mathrm{RoF}$ link nearly by
$20 \mathrm{~dB}$ in the regime of small-signal modulation and $10 \mathrm{~dB}$ under large-signal modulation. This substantial drop in the NF in spite of the small difference in the LF-RIN between the solitary laser modulated at $55.8 \mathrm{GHz}$ and the laser under $\mathrm{OFB}$, as shown in Fig. 5, is due to the corresponding improvement in the link gain $G_{\text {link }}$. It is worth to note that NF decreases with the increase in $G_{l} i n k$, as indicated in Eq. 8). The present calculations show that $G_{\text {link }}$ of the modulated laser under OFB ranges between $-24 \sim-5 \mathrm{~dB}$, which is $25 \mathrm{~dB}$ larger than the modulated solitary laser in the regime of small-signal modulation. The difference in $G_{\text {link }}$ decreases with the increase in $\mathrm{m}$ reaching $17 \mathrm{~dB}$ when $m=1.0$. In Figure 6, we examine the influence of the steady-state OFB coupling phase $\Delta \theta$ on the noise factor using the modulation responses given in Figure 2. At a given phase $\Delta \theta$, both the link gain $G_{\text {link }}$ and noise factor NF are calculated using the maximum modulation response and the corresponding $\mathrm{mm}$-wave frequency. The figure shows that NF increases with the increase in $\Delta \theta$; NF increases from 23 to $45 \mathrm{~dB}$ when $\Delta \theta$ increases from 0.8 to $1.45 \mathrm{rad} / \pi$.

\section{CONSLUSION}

We presented the enhancement of the intensity modulation response of semiconductor lasers subject to strong OFB over a passband beyond $50 \mathrm{GHz}$. We characterized the spectral characteristics of RIN of the laser in this ultra high-frequency band and assessed its contribution to the noise figure of a over 50 $\mathrm{GHz}-\mathrm{RiF}$ link. We compared the obtained findings with those of the solitary laser when modulated at both the same modulation frequency and modulation frequencies in the vicinity of the relaxation oscillation. We found that under strong OFB of $K_{e x}=1.45$, the modulation response decreases under the $-3 \mathrm{~dB}$ limit at frequencies lower than $f_{3 \mathrm{~dB}}$ of the solitary laser, and is enhanced again above $-3 \mathrm{~dB}$ over a band of $2 \mathrm{GHz}$ around the frequency of $55.8 \mathrm{GHz}$. The LF-RIN level increases very little with the increase in $m$ for both the laser under OFB and the solitary laser when modulated at $f_{m}=55.8 \mathrm{GHz}$, whereas the LF-RIN of the $15 \mathrm{GHz}$-solitary laser shows a considerable increase. We used the laser under OFB to design a $55.8 \mathrm{GHz}-$ RoF link and assessed the contribution of the laser RIN to NF of the link. NF was found to increase from 4 to $34 \mathrm{~dB}$ with the increase of the modulation depth $m$ from 0.05 to 1 . We showed that NF improves by using OFB nearly by $20 \mathrm{~dB}$ in the regime of small-signal modulation and $10 \mathrm{~dB}$ under large-signal modulation.

\section{ACKNOWLEDGEMENTS}

This work was funded by the Deanship of Scientific Research (DSR), King Abdulaziz University, under grant No. (8130/1433 HiCi). The authors, therefore, acknowledge technical and financial support of KAU.

\section{References}

[1] H. Ogawa, D. Polifko, and S. Banba, "Millimeter-wave fiber optics systems forpersonal radio communication“ IEEE Trans. Microwave Theory Tech. 40, 2285-2292 (1992). 
[2] S. C. Chan, S. K. Hwang, and J. M. Liu, "Period-one oscillation for photonicmicrowave transmission using an optically injected semiconductor laser“ Opti. Express 15, 14921-14935 (2007).

[3] S. Hwang, J. Liu, and J. White, " $35-\mathrm{CHz}$ intrinsic bandwidth for direct modulation in $1.3-\mu \mathrm{m}$ semiconductor lasers subject to strong injection locking“ IEEE Photon. Technol. Lett. 16, 972-974 (2004).

[4] S. C. Chan, S. K. Hwangb, and J. M. Liu, "Radio-over-fiber transmission from an optically injected semiconductor laser in period-one state“ Proc. of SPIE 6468 646811-646819 (2007).

[5] E. K. Lau, X. Zhao, H. K. Sung, D. Parekh, C. C. Hasnain, and M. C. Wu, "Strong optical injection-locked semiconductor lasers demonstrating $>100-\mathrm{GHz}$ resonance frequencies and $80-\mathrm{GHz}$ intrinsic bandwidths“ Opt. Express 16, 6609-6618 (2008).

[6] U. Feiste, "Optimization of modulation bandwidth of DBR lasers with detuned Bragg reflectors" IEEE J. Quantum Electron. 34, 2371-2379 (1998).

[7] G. Morthier, R. Schatz and 0. Kjebon, "Extended modulation bandwidth of DBR and external cavity lasers by utilizing a cavity resonance for equalization“ IEEE J. Quantum Electron. 36, 1468-1475 (2000).

[8] R. Mindaugas, A. Glitzky, U. Bandelow, M. Wolfrum. U. Troppenz, J. Kreissl, and W. Rehbein., "Improving the Modulation Bandwidth in Semiconductor Lasers by Passive Feedback" IEEE J. Sel. Top. Quantum Electron. 13 136-142 (2007).

[9] Y. Senlin, "Modulation Response Characteristics of an Optical Delayed Feedback Semiconductor Laser" in Proceedings to Symp. Photon. Optoelectron. 1-4 (Wuhan, 2011).

[10] J. S. Lawrence and D. M. Kane, "Nonlinear Dynamics of a Laser Diode With Optical Feedback Systems Subject to Modulation" IEEE J. Quantum Electron. 38, 185-192 (2002).

[11] U. Troppenz, J. Kreissl, W. Rehbein, and C. Bornholdt, "40Gbit/s directly modulated passive feedback laser" in Proceedings to Proc. 2oth Int. Conf. on Indium Phosphide and Related Materials 1-4 (Versailles, 2008).

[12] S. Weisser, E. C. Larkins, K. Czotscher, W. Benz, J. Daleiden, I. Esquivias, J. Fleissner et all., "Damping-limited modulation bandwidths up to $40 \mathrm{GHz}$ in undoped short-cavity Ino.35Cao.65AsGaAs multiple-quantum-well lasers" IEEE Photon. Technol. Lett. 8, 608-610 (1996).

[13] M. Ahmed, A. Bakry, R. Altuwirqi, M. Alghamdi and F. Koyama, "Enhancing modulation bandwidth of semiconductor lasers beyond $50 \mathrm{GHz}$ by strong optical feedback for use in mm-wave radio over fiber links“ Jap. J. Phys. Rap. Comm., accepted.

[14] M. Ahmed and M. Yamada, "Theoretical analysis of modecompetition noise in modulated laser diodes and its influence on noise performance of fiber links“ J. Phys. D 45, 405102 (2012).

[15] M. Ahmed, M. Yamada and M. Saito, "Numerical modeling of intensity and phase noise in semiconductor lasers" IEEE J. Quantum Electron. 37, 1600-1610 (2001).

[16] M. Ahmed, "Spectral lineshape and noise of semiconductor lasers under analog intensity modulation" J. Phys. D 41, 175104 (2008).

[17] N. Schunk and K. Petermann, "Numerical analysis of the feedback regimes for a single-mode semiconductor laser with external feedback“ IEEE J. Quantum Electron. QE 24, 1242-1247 (1988).
[18] K. Kallimiani and M. J. O'Mahony, "Relative intensity noise for laser diode with arbitrary amount of optical feedback" IEEE J. Quantum Electron. 34, 1438-1446 (1998).

[19] M. Ahmed and M. Yamada, "Field Fluctuations and Spectral Lineshape in Semiconductor Lasers Subjected to Optical Feedback" J. Appl. Phys. 95, 7573-7583 (2004).

[20] A. T. Ryan, G. P. Agrawal, R. Gray, C. Gage, "Optical feedback induced chaos and its control in multimode semiconductor lasers" IEEE J Quantum Electron. 30, 668-679 (1994).

[21] J. C. Yi, "High frequency modulation effects on the relative intensity noise properties of $405 \mathrm{~nm}$ InAlGaN laser diodes" Phys. Status solidi C 4, 1617-1620 (2007).

[22] M. Ahmed, N. Z. El-saysed and H. Ibrahim, "Chaos and noise control by current modulation in semiconductor lasers subject to optical feedback“ Eur. Phys. J. 66, 141 (2012).

[23] S. Abdulrhmann, M. Ahmed, T. Okamoto and M. Yamada, "An improved analysis of semiconductor laser dynamics under strong optical feedback“ IEEE J. Sel. Top. Quantum Electron. 9, 1265-1274 (2002).

[24] K. Sato, S. Kuwahar, and Y. Miyamoto, "Chirp Characteristics of 40-Gb/s Directly Modulated Distributed-Feedback Laser Diodes" J. Lightwave Technol. 23, 3790-3797 (2005).

[25] R. Lang and K. Kobayashi, "External optical feedback effects on semicobductor laser properties" IEEE J. Quantum Electron. QE 16, 347-355 (1980).

[26] M. Ahmed, "Numerical Approach to Field Fluctuations and Spec tral Lineshape in InGaAsP Laser Diodes" Int. J. Numer. Model. 17, 147-163 (2004)

[27] M. Ahmed, M. Yamada, and S. Abdulrhmann, “Numerical modeling of the route-to-chaos of semiconductor lasers under optical feedback and its dependence on the external-cavity length“ Int. J. Numer. Model. 22, 434-445 (2009).

[28] Y. H. Kao, N. M. Wang and H. M. Chen, "Mode description of routes to chaos in external-cavity coupled semiconductor lasers" IEEE J. Quantum Electron. 30, 1732-1739 (1994).

[29] H. Dalir and F. Koyamad, "Bandwidth enhancement of singlemode VCSEL with lateral optical feedback of slow light" IEICE Electron. Exp. 8, 1075-1081 (2011).

[30] C. Cox and W. S. Chang, RF photonic technology in optical fiber links (W. S. Chang, Cambridge Uni. Press, Cambridge, 2002).

[31] C. H. Cox III, Analog optical links (Cambridge Univ. Press, New York, 2004). 